\title{
Validation of the Accuracy of Self-Reported ABO Blood Types in the Japan Nurses' Health Study
}

\author{
Lobna Alkebsi' ${ }^{1}$ Hiroshi Ohnishi², Junko Nakajima-Shimada ${ }^{2}$, Yoko Onizuka², \\ Yuki Ideno ${ }^{3}$, Yasunori Sato ${ }^{4}$, Kunihiko Hayashi ${ }^{2 *}$
}

\begin{abstract}
Background: The associations between ABO blood type and risk of diseases including cancer have been reported from epidemiological studies. Self-reporting is one of the most widely used methods of collecting the ABO blood type information. Verifying the accuracy of self-reporting is important to consider measurement errors. We aimed to conduct validation of self-reported ABO blood types in the Japan Nurses' Health Study (JNHS), which is a large prospective cohort study. Methods: The concordance rate between self-reported and serologically or genetically inferred ABO blood groups was investigated for a subsample of 41 subjects from the Gunma Nurses' Health Study, which was a pilot cohort study that preceded the JNHS. The presence of antibodies to A or B antigens in serum (serological test) and allele types of the $\mathrm{ABO}$ gene (genotyping test) were determined by using frozen blood samples that were preserved for approximately 7 years. ABO blood types were determined from these tests and compared with self-reported data. Results: All of the nurses reported that their $\mathrm{ABO}$ blood groups were concordant with those determined by a serological and/or genotyping test. Self-reported ABO blood types of 35 of 38 (92.1\%) participants were consistent with the results from serological typing, while the answers of three participants were not. In these three participants, ABO genotypes that were inferred from genotyping of three single nucleotide polymorphisms in ABO loci perfectly matched with their self-reported ABO types, and all of these were O-type. Conclusions: Japanese health professionals report their blood type with a high degree of accuracy. Special attention should be paid to the O-type group in serological analysis of blood samples that have been preserved for several years in longitudinal studies.
\end{abstract}

Keywords: ABO blood-group system- self report- serological tests- genotyping techniques- blood preservation

Asian Pac J Cancer Prev, 20 (3), 789-793

\section{Introduction}

The associations between ABO blood type and risk of diseases, such as infectious diseases (Fry et al., 2008), type 2 diabetes (Qi et al., 2010), coronary heart disease (He et al., 2012), cancer (Zhang et al., 2014), and gastroduodenal ulcers (Alkebsi et al., 2018), have been reported from epidemiological studies. Information on the ABO blood type was obtained by a self-administered questionnaire in epidemiological studies in which a DNA genotyping test or serological test was not available. However, there have only been a few studies that assessed the validity of self-reported information compared with determination using a DNA genotyping test and serological test (Ito et al., 2001; Bider-Canfield Z and Cotterchio, 2014).

Blood samples are sometimes collected and preserved for many years in prospective cohort studies to allow for future nested case-control research using these preserved samples. In such studies, the ABO blood type may be determined using preserved blood samples by laboratory tests. Knowledge on the validity of the ABO blood type as determined by a DNA genotyping test or serological test for preserved samples is becoming increasingly important. However, the accuracy of these laboratory tests has not been well assessed in such settings compared with selfreported information.

The Japan Nurses' Health Study (JNHS) is a large prospective cohort study that investigated the effects of lifestyle and healthcare practices on the health of Japanese women (Hayashi et al., 2007). The participants of the JNHS were recruited from 2001 to 2007 . The feasibility of the research strategy of the JNHS was confirmed by a baseline survey pilot study that was started in 1999 called the Gunma Nurses' Health Study (GNHS) (Maeno et al., 2005; Kato et al., 2012). In previous studies, participants were required to report individual $\mathrm{ABO}$ blood types in a follow-up questionnaire. To evaluate the accuracy of

${ }^{1}$ Department of Haematology, Fukushima Medical University, Fukushima, ${ }^{2}$ Department of Laboratory Sciences, Graduate School of Health Sciences, Gunma University, ${ }^{3}$ Big Data Centre for Integrative Analysis, Gunma University Initiative for Advanced Research Maebashi, ${ }^{4}$ Department of Preventive Medicine and Public Health, Keio University School of Medicine, Tokyo, Japan. *For Correspondence: khayashi@gunma-u.ac.jp 
self-reported ABO blood types in the JNHS, we examined ABO serotypes and genotypes of 41 subjects from the GNHS by using preserved frozen blood samples. We compared the results with corresponding self-reported ABO blood types.

\section{Materials and Methods}

\section{Samples}

Between February and March 2008, peripheral blood samples were collected from 41 participants of the GNHS who provided written informed consent. Whole blood samples were collected in test tubes with or without ethylenediaminetetraacetic acid (EDTA). The samples without EDTA were incubated at room temperature for $30 \mathrm{~min}$ to $1 \mathrm{~h}$ and then centrifuged at $1400 \times \mathrm{g}$ for $20 \mathrm{~min}$ to remove blood clots. The resulting supernatant was used as serum samples. The samples with EDTA were used as whole blood samples without centrifugation. All of these samples were stored at $-80^{\circ} \mathrm{C}$ until later use. In December 2014, frozen serum and whole blood samples were thawed and examined for their $\mathrm{ABO}$ blood groups by serological and genotyping tests, respectively.

\section{Serological test: reverse $A B O$ grouping}

Reverse $\mathrm{ABO}$ grouping is a test for examining the presence of anti-A and/or anti-B antigen antibodies in serum. Briefly, two drops of serum sample were mixed with one drop of A-, B-, or O-type red blood cell (RBC) suspension ( $2-4 \%$ in saline) in a test tube. The tubes were gently shaken and centrifuged $(157 \times \mathrm{g}$ for $1 \mathrm{~min})$, and then agglutination was examined. If agglutination was observed only when the serum was mixed with A-type RBCs, this suggested the presence of specific antibodies to A blood type antigen, and the blood type of the sample was inferred as "B". Similarly, if agglutination was observed only when the serum was mixed with B-type RBCs, the sample blood type was inferred as " $\mathrm{A}$ ". Agglutination with A- and B-type RBCs and no agglutination suggested blood type "O" and "AB", respectively. O-type RBCs were used as a negative control. The serological test was performed by concealing information of self-reported $\mathrm{ABO}$ types and the results of the genotyping test.

\section{Extraction of genomic DNA from whole blood samples}

Genomic DNA was extracted from whole blood samples by using the TaqMan ${ }^{\circledR}$ Sample-to-SNPTM Kit (Applied Biosystems, Foster City, CA, USA) according to the manufacturer's protocol. The resulting genomic DNA solutions were thoroughly mixed and sored at $-80^{\circ} \mathrm{C}$ until later use.

Table 1. Estimation of ABO Blood Type with Three SNPs

\begin{tabular}{llccc}
\hline & & \multicolumn{3}{c}{ rs505922 } \\
& & TT & CT & CC \\
\hline \multirow{3}{*}{ rs8176746/rs8176747 } & AA/CC & ND & ND & BB \\
& AC/CG & ND & BO & AB \\
& CC/GG & OO & AO & AA \\
\hline
\end{tabular}

ND, not detected.

\section{Genotyping of $A B O$ blood groups}

The ABO gene on chromosome 9q34.2 codes glycosyltransferase, which transfers nucleotide donor sugars to the $\mathrm{H}$ antigen to form $\mathrm{ABO}$ blood group antigens (Yazer, 2005). Three single nucleotide polymorphisms (SNPs) on the ABO gene (rs8176746, rs8176747, and rs505922), which are highly responsible for ABO blood group phenotypes in the Japanese population, were analysed by direct nucleotide sequencing of polymerase chain reaction (PCR) products that included each SNP. $\mathrm{ABO}$ groups were determined by identification of specific combinations of the SNPs (Table 1) as shown by previous studies (Nakao et al., 2011; Tanikawa et al., 2012). Oligonucleotide sequences of primers for PCR are shown in Table 2. PCR was carried out on a T100 ${ }^{\mathrm{TM}}$ Thermal Cycler (Bio-Rad Laboratories Inc., Hercules, CA, USA) by using BIOTAQTM DNA polymerase (Bioline Reagents Ltd., London, UK) in $20 \mu$ l of reaction mixture according to the manufacturer's protocol. Amplification steps involved 35 cycles of $94^{\circ} \mathrm{C}$ for $30 \mathrm{~s}, 52^{\circ} \mathrm{C}$ for 45 $\mathrm{s}$, and $72^{\circ} \mathrm{C}$ for $45 \mathrm{~s}$ after a 3 -min preheating step at $94^{\circ} \mathrm{C}$. This was followed by a 2 -min post-incubation step at $72^{\circ} \mathrm{C}$. A volume of $5 \mu \mathrm{l}$ of reaction mixture was electrophoresed on a $2 \%$ agarose gel and PCR products were visualised by using GelRed (Biotium, Inc., Fremont, CA, USA) to confirm the size of the product and estimate its amount. The residual reaction mixture was then treated with $0.5 \mu \mathrm{l}$ of ExoSAP-ITTM PCR Product Cleanup (Affymetrix, Santa Clara, CA, USA) to remove excess primers and unincorporated nucleotides. DNA sequences of the PCR products were then analysed on the ABI 3130xl Prism Genetic Analyzer (Applied Biosystems) using the BigDye Terminator v1.1 Cycle Sequencing Kit (Applied Biosystems) and the BigDye XTerminator Purification Kit (Applied Biosystems).

Genotyping was performed by concealing information of self-reported $\mathrm{ABO}$ types and the results of the serological test. This study was approved $(\# 150,2012)$ by the Ethics Committee for Human Genome/Gene Analysis Research of Gunma University, Japan.

\section{Results}

The results of the reverse serological test of ABO blood groups, genotyping of ABO loci, and self-reported blood types are shown in Table 3 . Among the 41 participants, self-reported data were lacking for three participants (Nos. 1, 4, and 8) who did not answer the ABO blood type question or answered "unknown". Among the other participants, $\mathrm{ABO}$ phenotype frequencies in self-reported data were $42.1 \%$ for $\mathrm{A}, 18.4 \%$ for $\mathrm{B}, 10.5 \%$ for $\mathrm{AB}$, and $28.9 \%$ for $\mathrm{O}$.

Table 2. Sequences of Primer Sets for PCR for Analysis of Three SNPs in the ABO Loci

\begin{tabular}{ll}
\hline SNPs & \multicolumn{1}{c}{ Primer sequences (5'-3') } \\
\hline rs505922 & AAGGCTCCCTGACATGAGAC (forward) \\
& ACTTCCCGGGTCATTCTTTC (reverse) \\
rs8176746/ & GGAGGCCTTCACCTACGAG (forward) \\
rs8176747 & AGCTGCTGGTCCCACAAGTA (reverse) \\
\hline
\end{tabular}


Table 3. Self-Reported ABO Data and Serologically and Genetically Determined ABO Groups in a Subsample of 41 Subjects from the GNHS

\begin{tabular}{|c|c|c|c|c|c|}
\hline \multirow[t]{2}{*}{ Subject no. } & \multirow{2}{*}{$\begin{array}{l}\text { Self-reported } \\
\text { ABO }\end{array}$} & \multirow{2}{*}{$\begin{array}{c}\text { ABO } \\
\text { serotyping }\end{array}$} & \multicolumn{2}{|c|}{ SNPs in the ABO loci on chromosome $9 \mathrm{q} 34.2$} & \multirow{2}{*}{$\begin{array}{c}\text { ABO } \\
\text { genotyping }\end{array}$} \\
\hline & & & rs505922 & rs8176746/rs8176747 & \\
\hline 1 & $-*$ & $\mathrm{O}$ & TT & $\mathrm{CC} / \mathrm{GG}$ & $\mathrm{OO}$ \\
\hline $2 \dagger$ & $\mathrm{O}$ & $\mathrm{B}$ & $\mathrm{TT}$ & $\mathrm{CC} / \mathrm{GG}$ & $\mathrm{OO}$ \\
\hline 3 & $\mathrm{~B}$ & $\mathrm{~B}$ & $\mathrm{TT}$ & $\mathrm{AC} / \mathrm{CG}$ & undetermined \\
\hline 4 & $-*$ & $\mathrm{~B}$ & $\mathrm{CT}$ & $\mathrm{AC} / \mathrm{CG}$ & $\mathrm{BO}$ \\
\hline 5 & A & A & $\mathrm{CT}$ & $\mathrm{CC} / \mathrm{GG}$ & $\mathrm{AO}$ \\
\hline 6 & $\mathrm{~B}$ & $\mathrm{~B}$ & $\mathrm{CT}$ & $\mathrm{AC} / \mathrm{CG}$ & $\mathrm{BO}$ \\
\hline 7 & A & A & $\mathrm{CC}$ & $\mathrm{CC} / \mathrm{GG}$ & AA \\
\hline 8 & $-*$ & A & $\mathrm{TT}$ & $\mathrm{CC} / \mathrm{GG}$ & $\mathrm{OO}$ \\
\hline 9 & A & A & $\mathrm{CC}$ & $\mathrm{CC} / \mathrm{GG}$ & AA \\
\hline 10 & A & A & $\mathrm{CC}$ & $\mathrm{CC} / \mathrm{GG}$ & $\mathrm{AA}$ \\
\hline 11 & $\mathrm{O}$ & $\mathrm{O}$ & $\mathrm{TT}$ & $\mathrm{CC} / \mathrm{GG}$ & $\mathrm{OO}$ \\
\hline 12 & A & A & $\mathrm{CT}$ & $\mathrm{CC} / \mathrm{GG}$ & $\mathrm{AO}$ \\
\hline $13 \dagger$ & $\mathrm{O}$ & A & $\mathrm{TT}$ & $\mathrm{CC} / \mathrm{GG}$ & $\mathrm{OO}$ \\
\hline 14 & $\mathrm{AB}$ & $\mathrm{AB}$ & $\mathrm{CC}$ & $\mathrm{AC} / \mathrm{CG}$ & $\mathrm{AB}$ \\
\hline 15 & $\mathrm{O}$ & $\mathrm{O}$ & $\mathrm{TT}$ & $\mathrm{CC} / \mathrm{GG}$ & $\mathrm{OO}$ \\
\hline 16 & A & A & $\mathrm{CT}$ & $\mathrm{CC} / \mathrm{GG}$ & $\mathrm{AO}$ \\
\hline $17 \dagger$ & $\mathrm{AB}$ & $\mathrm{AB}$ & $\mathrm{CC}$ & $\mathrm{CC} / \mathrm{GG}$ & AA \\
\hline 18 & A & A & $\mathrm{CC}$ & $\mathrm{CC} / \mathrm{GG}$ & AA \\
\hline 19 & $\mathrm{O}$ & $\mathrm{O}$ & $\mathrm{TT}$ & $\mathrm{CC} / \mathrm{GG}$ & $\mathrm{OO}$ \\
\hline 20 & A & A & $\mathrm{CT}$ & CCGG & $\mathrm{AO}$ \\
\hline 21 & B & B & $\mathrm{CT}$ & $\mathrm{AC} / \mathrm{CG}$ & $\mathrm{BO}$ \\
\hline 22 & A & A & $\mathrm{CT}$ & $\mathrm{CC} / \mathrm{GG}$ & $\mathrm{AO}$ \\
\hline 23 & $\mathrm{AB}$ & $\mathrm{AB}$ & $\mathrm{CC}$ & $\mathrm{AC} / \mathrm{CG}$ & $\mathrm{AB}$ \\
\hline 24 & B & B & $\mathrm{CT}$ & $\mathrm{AC} / \mathrm{CG}$ & BO \\
\hline 25 & A & A & $\mathrm{CC}$ & $\mathrm{CC} / \mathrm{GG}$ & AA \\
\hline 26 & A & A & $\mathrm{CT}$ & $\mathrm{CC} / \mathrm{GG}$ & $\mathrm{AO}$ \\
\hline 27 & A & A & $\mathrm{CC}$ & $\mathrm{CC} / \mathrm{GG}$ & AA \\
\hline 28 & B & B & $\mathrm{CT}$ & $\mathrm{AC} / \mathrm{CG}$ & $\mathrm{BO}$ \\
\hline 29 & $\mathrm{AB}$ & $\mathrm{AB}$ & $\mathrm{CC}$ & $\mathrm{AC} / \mathrm{CG}$ & $\mathrm{AB}$ \\
\hline 30 & A & A & $\mathrm{CT}$ & $\mathrm{CC} / \mathrm{GG}$ & $\mathrm{AO}$ \\
\hline 31 & B & B & $\mathrm{CT}$ & $\mathrm{AC} / \mathrm{CG}$ & $\mathrm{BO}$ \\
\hline 32 & A & A & $\mathrm{CT}$ & $\mathrm{CC} / \mathrm{GG}$ & $\mathrm{AO}$ \\
\hline 33 & $\mathrm{O}$ & $\mathrm{O}$ & $\mathrm{TT}$ & $\mathrm{CC} / \mathrm{GG}$ & $\mathrm{OO}$ \\
\hline 34 & $\mathrm{O}$ & $\mathrm{O}$ & TT & $\mathrm{CC} / \mathrm{GG}$ & $\mathrm{OO}$ \\
\hline 35 & $\mathrm{O}$ & $\mathrm{O}$ & $\mathrm{TT}$ & $\mathrm{CC} / \mathrm{GG}$ & $\mathrm{OO}$ \\
\hline 36 & B & B & $\mathrm{CC}$ & $\mathrm{AA} / \mathrm{CC}$ & $\mathrm{BB}$ \\
\hline $37 \dagger$ & $\mathrm{O}$ & A & $\mathrm{TT}$ & $\mathrm{CC} / \mathrm{GG}$ & $\mathrm{OO}$ \\
\hline 38 & $\mathrm{~A}$ & A & $\mathrm{CT}$ & $\mathrm{CC} / \mathrm{GG}$ & $\mathrm{AO}$ \\
\hline 39 & A & A & $\mathrm{CT}$ & $\mathrm{CC} / \mathrm{GG}$ & $\mathrm{AO}$ \\
\hline 40 & $\mathrm{O}$ & $\mathrm{O}$ & $\mathrm{TT}$ & $\mathrm{CC} / \mathrm{GG}$ & $\mathrm{OO}$ \\
\hline 41 & $\mathrm{O}$ & $\mathrm{O}$ & TT & $\mathrm{CC} / \mathrm{GG}$ & $\mathrm{OO}$ \\
\hline
\end{tabular}

*, Participants did not answer the ABO blood type question or answered "unknown"; $\uparrow$, Inconsistency among the self-reported data, serotyping, and genotyping.

Self-reported ABO blood types of 35 of 38 (92.1\%) participants were consistent with the results from serological typing, while the answers of three participants (Nos. 2, 13, and 37) were not. In these three participants, $\mathrm{ABO}$ genotypes that were inferred from genotyping of three SNPs in ABO loci ( $r s 8176746, r 8176747$, and rs505922) perfectly matched their self-reported $\mathrm{ABO}$ types. Genotyping data showed that self-reported ABO blood types of two (Nos. 3 and 17) of 38 participants were inconsistent with the genetically inferred blood groups. 
For sample No. 3, the combination of three ABO SNPs did not match any of the reported patterns of ABO groups. For sample No. 17, genotyping results showed blood type A, but the self-reported and serologically confirmed ABO types were AB. Therefore, self-reported ABO types of 36 of 38 (94.7\%) participants were consistent with the genotyping results. Except for participant No. 3, the determined allele frequencies among the participants were $33.8 \%$ for the A allele, $13.8 \%$ for the $\mathrm{B}$ allele, and $52.5 \%$ for the $\mathrm{O}$ allele. Genetically determined ABO group frequencies were well matched with those expected from allele frequencies under Hardy-Weinberg equilibrium $(p=0.53$, chi-square test), which ensured quality of the genotyping data.

\section{Discussion}

This study examined the accuracy of self-reported ABO blood types of a large prospective cohort study, the JNHS, by using subsamples from a pilot study (GNHS). Self-reported ABO blood types were compared with laboratory analysis of blood samples that had been preserved for several years from the GNHS study. ABO phenotype frequencies in self-reported data were similar to those observed in a previous report of Japanese populations (Fujita et al., 1978). All of the nurses reported that their ABO blood groups were concordant with those determined by a serological and/or genotyping test. The self-reported ABO blood types were highly consistent with serologically (92.1\%) and genetically (94.7\%) reconfirmed blood types. Overall, our results suggest that self-reported $\mathrm{ABO}$ blood type data are reliable in the JNHS.

The ABO blood group is one of the most important systems in DNA profiling, as well as in transfusion medicine. Furthermore, many studies have suggested that the ABO blood type is associated with various diseases. Using self-reported data, our previous epidemiological study of Japanese women showed that women with blood type $\mathrm{O}$ have an increased susceptibility to gastroduodenal ulceration (Alkebsi et al., 2018). Therefore, accuracy of the self-reported ABO blood type is important for evaluating the association of genetic factors with diseases in such a study. In the JNHS, all participants were women working in the health profession. The JNHS participants have a greater understanding about the importance of personal biological characteristics that affect health and medical issues compared with the general population. Such a condition likely contributed to the high accuracy of the self-reported ABO type data in the JNHS.

In our experiments, self-reported data from three subjects (Nos. 2, 13, 37) were inconsistent with the results of a serological test. In contrast, the self-reported ABO types for these participants were perfectly matched with the genotyping data, and all of these were O-type. Therefore, self-reported "O" is correct, and a low accuracy for detecting O-type blood might be a disadvantage of our serological test. In this study, frozen blood samples, which were preserved from 2008 to 2014, were thawed and subjected to serological analysis. Such a long preservation and freeze-thaw treatment, at least in part, might induce inactivation of serum antibodies. If this is the case, such a condition could lower the accuracy of analysis, especially for detecting O-type blood. This is because the serological test requires two antigen-antibody reactions for A- and B-type RBCs. Therefore, genetic ABO typing could be more reliable than a serological test for frozen blood samples. Similarly, DNA-based methods provide more reliable information than the traditional serological method in individuals, especially regarding transfusion medicine and immunohaematology disorders (Keller, 2015; Belsito et al., 2017). However, the ABO genotype of one sample (No. 3) in our study could not be determined. Additionally, another sample (No. 17, AA) did not match the self-reported $(\mathrm{AB})$ and serologically determined $\mathrm{ABO}$ blood type (AB). Analyses of additional SNPs are required for more accurate determination of the ABO blood type.

In conclusion, this study shows that self-reported ABO blood types in JNHS are mostly accurate, suggesting that they are useful for epidemiological surveys. Special attention should be paid to the O-type group in serological analysis of blood samples that have been preserved for several years in longitudinal studies. Confirming $\mathrm{ABO}$ determination by laboratory analysis in cases of preserved blood samples with self-reported ABO blood type information may be useful.

\section{Funding}

This work was partly supported by the Japan Society for the Promotion of Science Grants-in-Aid for Scientific Research (B) (grant number: JP24501367).

\section{Conflict of interest}

None declared.

\section{Acknowledgements}

The authors would like to thank the JNHS participants for their contribution to this research. We would also like to thank Ms. Eriko Urano for technical support.

\section{References}

Alkebsi L, Ideno Y, Lee JS, et al (2018). Gastroduodenal ulcers and ABO blood group: the Japan Nurses' Health Study (JNHS). J Epidemiol, 28, 34-40.

Belsito A, Magnussen K, Napoli C (2017). Emerging strategies of blood group genotyping for patients with hemoglobinopathies. Transfus Apher Sci, 56, 206-13.

Bider-Canfield Z, Cotterchio M (2014). Self-reported ABO blood type compared with DNA-derived blood group. Epidemiology, 25, 936-7.

Fry AE, Griffiths MJ, Auburn S, et al (2008). Common variation in the $\mathrm{ABO}$ glycosyltransferase is associated with susceptibility to severe Plasmodium falciparum malaria. Hum Mol Genet, 17, 567-76.

Fujita Y, Tanimura M, Tanaka K (1978). The distribution of the ABO blood groups in Japan. Jinrui Idengaku Zasshi, 23, 63-109.

Hayashi K, Mizunuma H, Fujita T, et al (2007). Design of the Japan Nurses' Health Study: a prospective occupational cohort study of women's health in Japan. Ind Health, 45, 679-686.

He M, Wolpin B, Rexrode K, et al (2012). ABO blood group 
and risk of coronary heart disease in two prospective cohort studies. Arterioscler Thromb Vasc Biol, 32, 2314-20.

Ito H, Matsuo K, Saito T (2001). Valid responses to ABO blood type question in self-reporting questionnaire. Asian Pac $J$ Cancer Prev, 2, 315-7.

Kato C, Shimada J, Hayashi K (2012). Sleepiness during shift work in Japanese nurses: A comparison study using JESS, SSS, and actigraphy. Sleep Biological Rhythms, 10, 109-17.

Keller MA (2015). The role of red cell genotyping in transfusion medicine. Immunohematology, 31, 49-52.

Maeno T, Ohta A, Hayashi K, et al (2005). Impact of reproductive experience on women's smoking behaviour in Japanese nurses. Public Health, 119, 816-24.

Nakao M, Matsuo K, Hosono S, et al (2011). ABO blood group alleles and the risk of pancreatic cancer in a Japanese population. Cancer Sci, 102, 1076-80.

Qi L, Cornelis MC, Kraft P, et al (2010). Genetic variants at 2 q24 are associated with susceptibility to type 2 diabetes. Hum Mol Genet, 19, 2706-15.

Tanikawa C, Urabe Y, Matsuo K, et al (2012). A genome-wide association study identifies two susceptibility loci for duodenal ulcer in the Japanese population. Nat Genet, 44, 430-34.

Yazer MH (2005). What a difference 2 nucleotides make: a short review of ABO genetics. Transfus Med Rev, 19, 200-9.

Zhang BL, He N, Huang YB, et al (2014). ABO blood groups and risk of cancer: a systematic review and meta-analysis. Asian Pac J Cancer Prev, 15, 4643-50.

\section{cc) (i) (8)}

This work is licensed under a Creative Commons AttributionNon Commercial 4.0 International License. 